PROCEEDINGS OF THE AMERICAN MATHEMATICAL SOCIETY

Volume 124, Number 8, August 1996

\title{
EXTREMAL PROBLEMS IN MINKOWSKI SPACE RELATED TO MINIMAL NETWORKS
}

\author{
K. J. SWANEPOEL
}

(Communicated by Peter Li)

\begin{abstract}
We solve the following problem of Z. Füredi, J. C. Lagarias and F. Morgan (1991): Is there an upper-bound polynomial in $n$ for the largest cardinality of a set $S$ of unit vectors in an $n$-dimensional Minkowski space (or Banach space) such that the sum of any subset has norm less than 1 ? We prove that $|S| \leq 2 n$ and that equality holds iff the space is linearly isometric to $\ell_{\infty}^{n}$, the space with an $n$-cube as unit ball. We also remark on similar questions they raised that arose out of the study of singularities in length-minimizing networks in Minkowski spaces.
\end{abstract}

\section{INTRODUCTION}

In [LM] Lawlor and Morgan derived a geometrical description for the singularities (Steiner points) of a length-minimizing network connecting a finite set of points in a smooth Minkowski space (finite-dimensional Banach space). In Euclidean space the geometrical description is equivalent to the classical result that at a singularity three line segments meet at $120^{\circ}$ angles. See also $[\mathrm{BG}],[\mathrm{M}]$ and $[\mathrm{CR}]$ for a discussion of length-minimizing networks and their history. The geometrical description of Lawlor and Morgan leads to extremal problems of a combinatorial type in strictly convex Minkowski spaces. Such problems are considered in [FLM]. In this note we briefly remark on some of these problems and solve one of the open problems stated in [FLM] (see Theorem 3).

\section{Preliminaries}

We denote the real numbers by $\mathbb{R}$ and the real vector space of $n$-tuples of real numbers by $\mathbb{R}^{n}$. The coordinates of a vector $\mathbf{x} \in \mathbb{R}^{n}$ will be denoted by $\mathbf{x}=$ $(\mathbf{x}(1), \mathbf{x}(2), \ldots, \mathbf{x}(n))$. The standard basis $\mathbf{e}_{1}, \mathbf{e}_{2}, \ldots, \mathbf{e}_{n}$ will be used, where $\mathbf{e}_{i}$ is the vector for which $\mathbf{e}_{i}(i)=1$ and $\mathbf{e}_{i}(j)=0$ for $i \neq j$. A Minkowski space (or finitedimensional Banach space) $\left(\mathbb{R}^{n}, \Phi\right)$ is $\mathbb{R}^{n}$ endowed with a norm $\Phi$. A Minkowski space is strictly convex if $\Phi(\mathbf{x})=\Phi(\mathbf{y})=1, \mathbf{x} \neq \mathbf{y}$ implies $\Phi(\mathbf{x}+\mathbf{y})<2$.

Received by the editors February 21, 1995.

1991 Mathematics Subject Classification. Primary 52A40, 52A21, 49F10.

Key words and phrases. Minimal networks, Minkowski spaces, finite-dimensional Banach spaces, sums of unit vectors problem.

(C)1996 American Mathematical Society 
We denote by $\ell_{p}^{n}$ the $n$-dimensional Minkowski space with norm

$$
\Phi_{p}(\mathbf{x})=\left(\sum_{i=1}^{n}|\mathbf{x}(i)|^{p}\right)^{1 / p}
$$

for $p \geq 1$, and by $\ell_{\infty}^{n}$ the space with norm

$$
\Phi_{\infty}(\mathbf{x})=\max _{1 \leq i \leq n}|\mathbf{x}(i)| .
$$

We now state Auerbach's Lemma which relates the spaces $\ell_{1}^{n}$ and $\ell_{\infty}^{n}$ to an arbitrary Minkowski space in $n$ dimensions. A proof may be found in [Pi, page 29].

Auerbach's Lemma. For any Minkowski space $\left(\mathbb{R}^{n}, \Phi\right)$ there exists a linear isomorphism $T: \mathbb{R}^{n} \rightarrow \mathbb{R}^{n}$ such that $\Phi_{\infty}(\mathbf{x}) \leq \Phi(T \mathbf{x}) \leq \Phi_{1}(\mathbf{x})$, i.e.

$$
\max _{1 \leq i \leq n}|\mathbf{x}(i)| \leq \Phi(T \mathbf{x}) \leq \sum_{i=1}^{n}|\mathbf{x}(i)| .
$$

We denote the $n$-dimensional Lebesgue measure (or volume) of measurable $V \subseteq$ $\mathbb{R}^{n}$ by $\operatorname{vol}(V)$. If $U, V \subseteq \mathbb{R}^{n}$, then we define $U+V=\{\mathbf{u}+\mathbf{v} \mid \mathbf{u} \in U, \mathbf{v} \in V\}$. The Brunn-Minkowski inequality relates the volumes of compact $U$ and $V$ to that of $U+V$. A proof may be found in [BZ].

Brunn-Minkowski inequality. If $U, V \subseteq \mathbb{R}^{n}$ are compact, then

$$
(\operatorname{vol}(U+V))^{1 / n} \geq(\operatorname{vol}(U))^{1 / n}+(\operatorname{vol}(V))^{1 / n} .
$$

\section{Extremal PRoblems}

From now on $S$ will denote a finite set of unit vectors in a Minkowski space. In [FLM] the following type of extremal problems is considered: Find the largest cardinality of $S$ satisfying a selection of the following conditions:

$$
\begin{array}{cl}
\Phi\left(\sum_{\mathbf{x} \in J} \mathbf{x}\right) \leq 1 \text { for all } J \subseteq S & \text { (the strong collapsing condition), } \\
\Phi(\mathbf{x}+\mathbf{y}) \leq 1 \text { for all } \mathbf{x}, \mathbf{y} \in S, \mathbf{x} \neq \mathbf{y} & \text { (the weak collapsing condition), } \\
\sum_{\mathbf{x} \in S} \mathbf{x}=\mathbf{0} & \text { (the strong balancing condition), } \\
\begin{array}{l}
\mathbf{0} \text { is in the relative interior } \\
\text { of the convex hull of } S
\end{array} & \text { (the weak balancing condition). }
\end{array}
$$

See $[\mathrm{LM}]$ and $[\mathrm{FLM}]$ for the connection between these conditions and minimal networks. In [FLM] it is proved that $\left(A^{\prime}\right)$ and $\left(B^{\prime}\right)$ together give an upper bound $|S| \leq 2 n$ for an arbitrary Minkowski space, and $|S| \leq n+1$ for strictly convex Minkowski spaces. In $[\mathrm{LM}]$ it is proved that there exist a strictly convex norm on $\mathbb{R}^{n}$ and a subset $S$ of $n+1$ unit vectors satisfying $(A)$ and $(B) .|S|=2 n$ is attained in, for example, $\ell_{\infty}^{n}$ with $S=\left\{ \pm \mathbf{e}_{i} \mid 1 \leq i \leq n\right\}$, in which case even the strong conditions $(A)$ and $(B)$ hold. However, there are other Minkowski spaces where equality is also attained (see Theorem 1 ). This is to be contrasted with Theorem 3 , where we show that the extreme case for $S$ satisfying $(A)$ and $(B)$ can only be attained for $\ell_{\infty}^{n}$.

Theorem 1. For infinitely many $n \geq 1$ there exists a set of unit vectors $S=$ $\left\{\mathbf{x}_{1}, \ldots, \mathbf{x}_{2 n}\right\} \subseteq \ell_{1}^{n}$ satisfying $\left(A^{\prime}\right)$ and the strong balancing condition $(B)$. In particular, such a set exists if a Hadamard matrix of order $n$ exists. 
Proof. We recall that an $n \times n$ Hadamard matrix $H$ consists of $( \pm 1)$-entries such that $H H^{t}=n I$, and such matrices exist for infinitely many $n$ (see [vLW, Chapter 18]). We let $\mathbf{v}_{1}, \ldots, \mathbf{v}_{n}$ be the column vectors of $H$, and set $\mathbf{x}_{i}=\frac{1}{n} \mathbf{v}_{i}$ for $i=1, \ldots, n$. Then $S:=\left\{ \pm \mathbf{x}_{i} \mid 1 \leq i \leq n\right\}$ is a set of $2 n$ unit vectors. Since the column vectors of $H$ are orthogonal, $\left\langle\mathbf{v}_{i}, \mathbf{v}_{j}\right\rangle=0$ for $i \neq j$, implying that $\Phi_{1}\left(\mathbf{x}_{i}+\mathbf{x}_{j}\right)=1$ and $\Phi_{1}\left(\mathbf{x}_{i}-\mathbf{x}_{j}\right)=1$ for all $i \neq j$. It follows that $S$ satisfies $\left(A^{\prime}\right)$ and $(B)$.

The question now is what happens if there is no balancing condition present. In [FLM] an upper bound of $|S|<3^{n}$ is derived from the weak collapsing condition $\left(A^{\prime}\right)$ alone using a volume argument. Using the Brunn-Minkowski inequality we obtain a sharper bound (Theorem 2). In [FLM] a strictly convex norm and a set $S$ of unit vectors with $|S| \geq(1.02)^{n}$ satisfying $\left(A^{\prime}\right)$ are constructed for all sufficiently large $n$. It would be interesting to find the greatest lower bound of the $\alpha$ 's for which $|S| \leq \alpha^{n}$ for any set $S$ of unit vectors in an arbitrary Minkowski space satisfying $\left(A^{\prime}\right)$, and sufficiently large $n$.

Theorem 2. If a set $S$ of unit vectors in $\mathbb{R}^{n}$ satisfies $\left(A^{\prime}\right)$, then $|S|<2^{n+1}$.

Proof. We denote the closed unit ball with centre $\mathbf{x}$ and radius $r$ by $B(\mathbf{x}, r)=$ $\left\{\mathbf{y} \in \mathbb{R}^{n} \mid \Phi(\mathbf{x}-\mathbf{y}) \leq r\right\}$, and the volume of a ball of unit radius by $\beta$. For distinct $\mathbf{x}, \mathbf{y} \in S$ we obtain from the triangle inequality that $\Phi(\mathbf{x}-\mathbf{y}) \geq 1$. Let $k=|S|$. We partition $S$ into two sets $S_{1}$ and $S_{2}$ of sizes $\lfloor k / 2\rfloor$ and $\lceil k / 2\rceil$, respectively. Let $V_{i}=B\left(\mathbf{0}, \frac{1}{2}\right) \cup \bigcup_{\mathbf{x} \in S_{i}} B\left(\mathbf{x}, \frac{1}{2}\right)$ for $i=1,2$. Clearly, each $V_{i}$ consists of closed balls with disjoint interiors, and therefore, $\operatorname{vol}\left(V_{1}\right)=\beta(\lfloor k / 2\rfloor+1) 2^{-n}$ and $\operatorname{vol}\left(V_{2}\right)=$ $\beta(\lceil k / 2\rceil+1) 2^{-n}$. Using $\left(A^{\prime}\right)$ we obtain $V_{1}+V_{2} \subseteq B(\mathbf{0}, 2)$ and $\operatorname{vol}\left(V_{1}+V_{2}\right) \leq 2^{n} \beta$. By the Brunn-Minkowski inequality we now have

$$
2 \beta^{1 / n} \geq \frac{1}{2} \beta^{1 / n}(\lfloor k / 2\rfloor+1)^{1 / n}+\frac{1}{2} \beta(\lceil k / 2\rceil+1)^{1 / n}>\beta^{1 / n}(k / 2)^{1 / n}
$$

and $|S|<2^{n+1}$.

From the above proof we actually find that if $\Phi(\mathbf{x})=\Phi(\mathbf{y})=1$ and $\Phi(\mathbf{x}+\mathbf{y}) \leq 1$ imply $\Phi(\mathbf{x}-\mathbf{y}) \geq r>1$, then $|S| \leq 2(1+1 / r)^{n}+1$ for $S$ satisfying $\left(A^{\prime}\right)$. Such is the case for $\ell_{p}^{n}$ : It follows from the Clarkson inequality [C] for $p \geq 2$, and the Hanner inequality [H] for $1<p<2$, that $r$ may be taken to be $3^{1 / p}$ for $p \geq 2$, and $\left(2^{p}-1\right)^{1 / p}$ for $1<p<2$.

For $\ell_{1}^{n}$ an upper bound $|S| \leq 2^{n}$ holds: If the coordinates of two unit vectors $\mathbf{x}$ and $\mathbf{y}$ have the same sequence of signs, i.e. $\operatorname{sgn}(\mathbf{x}(i))=\operatorname{sgn}(\mathbf{y}(i))$ for all $i=1, \ldots, n$, then $\Phi_{1}(\mathbf{x}+\mathbf{y})=2$, contradicting $\left(A^{\prime}\right)$. In the Euclidean case $\ell_{2}^{n}$ we of course have $|S| \leq 3$, independent of $n$. For $\ell_{\infty}^{n}$ the sharp upper bound $|S| \leq 2 n$ holds: If $|S| \geq 2 n+1$, then by the pigeon-hole principle there are three vectors $\mathbf{x}, \mathbf{y}, \mathbf{z} \in S$ and an $i \in\{1, \ldots, n\}$ such that $|\mathbf{x}(i)|=|\mathbf{y}(i)|=|\mathbf{z}(i)|=1$. Some two of these vectors will have the same sign in the $i$ th coordinate, and their sum will then have a norm of 2 .

In [FLM, Problem 3.7] the question is asked whether the strong collapsing condition $(A)$ on its own gives an upper bound for $|S|$ that is polynomial in $n$. A linear upper bound may be derived by the same technique as in Theorem 2 . We partition the elements of $S$ except for at most 2 into subsets $S_{1}, \ldots, S_{k}$ of size 3 , where $k=\lfloor|S| / 3\rfloor$. For $i=1, \ldots, k$ let

$$
V_{i}=\bigcup_{\mathbf{x} \in S_{i}} B\left(\mathbf{x}, \frac{1}{2}\right) \cup \bigcup_{\mathbf{x}, \mathbf{y} \in S_{i}, \mathbf{x} \neq \mathbf{y}} B\left(\mathbf{x}+\mathbf{y}, \frac{1}{2}\right) .
$$


From $(A)$ it follows that each $V_{i}$ consists of 6 balls with disjoint interiors and $V_{1}+\cdots+V_{k} \subseteq B\left(\mathbf{0}, \frac{1}{2} k+1\right)$. By the Brunn-Minkowski inequality we obtain $\frac{1}{2} k+1 \geq \frac{1}{2} 6^{1 / n} k$ and $k \leq 2 /\left(6^{1 / n}-1\right)$. Therefore, $|S| \leq 6 /\left(6^{1 / n}-1\right)+2<(6 / \ln 6) n$, after some calculus. This bound is not sharp, however. In the following theorem we derive the sharp upper bound $|S| \leq 2 n$.

Theorem 3. Let $S$ be a finite set of unit vectors in a Minkowski space $\left(\mathbb{R}^{n}, \Phi\right)$ satisfying the collapsing condition $(A)$. Then $|S| \leq 2 n$, and equality holds iff $\left(\mathbb{R}^{n}, \Phi\right)$ is linearly isometric to $\ell_{\infty}^{n}$, with $S$ corresponding to the set $\left\{ \pm \mathbf{e}_{i} \mid 1 \leq i \leq n\right\}$ under any isometry.

Proof. By Auerbach's Lemma we may assume (after applying a linear isomorphism of $\mathbb{R}^{n}$ ) that for any vector $\mathbf{x} \in \mathbb{R}^{n}$ the inequalities (1) hold, with $T$ now the identity. Choose $m$ distinct vectors $\mathbf{x}_{1}, \ldots, \mathbf{x}_{m}$ from $S$. By (1) we have

$$
\sum_{i=1}^{n}\left|\mathbf{x}_{j}(i)\right| \geq 1 \text { for all } j=1, \ldots, m .
$$

Suppose that for some coordinate $i \in\{1, \ldots, n\}$ we have

$$
\sum_{\substack{j=1 \\ \mathbf{x}_{j}(i) \geq 0}}^{n} \mathbf{x}_{j}(i)>1 .
$$

Then

$$
\Phi\left(\sum_{\substack{j=1 \\ \mathbf{x}_{j}(i) \geq 0}}^{m} \mathbf{x}_{j}\right) \geq \sum_{\substack{j=1 \\ \mathbf{x}_{j}(i) \geq 0}}^{m} \mathbf{x}_{j}(i)
$$

by (1), contradicting $(A)$. Therefore,

$$
\sum_{\substack{j=1 \\ \mathbf{x}_{j}(i) \geq 0}}^{m} \mathbf{x}_{j}(i) \leq 1 \text { for all } i=1, \ldots, n,
$$

and similarly,

$$
\sum_{\substack{j=1 \\ \mathbf{x}_{j}(i) \leq 0}}^{m}-\mathbf{x}_{j}(i) \leq 1 \text { for all } i=1, \ldots, n .
$$

From (3) and (4) it follows that $\sum_{j=1}^{m}\left|\mathbf{x}_{j}(i)\right| \leq 2$ for all $i=1, \ldots, n$, and from (2) we have

$$
m \leq \sum_{j=1}^{m} \sum_{i=1}^{n}\left|\mathbf{x}_{j}(i)\right| \leq 2 n
$$

and $|S| \leq 2 n$.

If $|S|=2 n$ for some set of unit vectors $S=\left\{\mathbf{x}_{1}, \ldots, \mathbf{x}_{2 n}\right\}$ satisfying $(A)$, then equality must hold in (5), (3) and (4). Therefore, $\sum_{j=1}^{2 n} \mathbf{x}_{j}=0$, showing that in the extreme case the strong balancing condition $(B)$ must be satisfied. We now show that conditions $(A)$ and $(B)$ together with the assumption $|S|=2 n$ imply that $\left(\mathbb{R}^{n}, \Phi\right)$ is linearly isometric to $\ell_{\infty}^{n}$, and $S$ corresponds to $\left\{ \pm \mathbf{e}_{i} \mid 1 \leq i \leq n\right\}$, as claimed in [FLM].

We recall Theorem 3.1 of [FLM]: 
If $\left(\mathbb{R}^{n}, \Phi\right)$ is a Minkowski space and $S$ is a set of unit vectors satisfying $\left(A^{\prime}\right)$ and $\left(B^{\prime}\right)$, then $|S| \leq 2 n$, and if equality holds, then $S$ corresponds to $\left\{ \pm \mathbf{e}_{i} \mid 1 \leq i \leq n\right\}$ under some linear isomorphism.

We therefore have $S=\left\{ \pm \mathbf{x}_{i} \mid i=1 \leq i \leq n\right\}$, where the $\mathbf{x}_{i}$ 's are linearly independent. We first show that if $\left(\mathbb{R}^{n}, \Phi\right)=\ell_{\infty}^{n}$, then $S=\left\{ \pm \mathbf{e}_{i} \mid 1 \leq i \leq n\right\}$ must hold. For $i=1, \ldots, n$ choose $j_{i} \in\{1, \ldots, n\}$ such that $\left|\mathbf{x}_{i}\left(j_{i}\right)\right|=1$. After renaming, we may assume $\mathbf{x}_{i}\left(j_{i}\right)=1$. The $j_{i}$ 's must be distinct, otherwise $(A)$ is contradicted. We may therefore rename the $\mathbf{x}_{i}$ 's to obtain $\mathbf{x}_{i}(i)=1$ for $i=1, \ldots, n$. If we have $\mathbf{x}_{i}(j) \neq 0$ for some $i \neq j$, then either $\Phi_{\infty}\left(\mathbf{x}_{i}+\mathbf{x}_{j}\right)>1$ or $\Phi_{\infty}\left(-\mathbf{x}_{i}+\mathbf{x}_{j}\right)>1$, contradicting $(A)$. Therefore, $\mathbf{x}_{i}(j)=0$ for all $i \neq j$, and we have $S=\left\{ \pm \mathbf{e}_{i} \mid i=1, \ldots, n\right\}$.

To show that in fact $\left(\mathbb{R}^{n}, \Phi\right)$ is linearly isometric to $\ell_{\infty}^{n}$, we use the following theorem of Petty [Pe] (see also [FLM, Theorem 2.1]):

If $T$ is a subset of a Minkowski space $\left(\mathbb{R}^{n}, \Phi\right)$ such that $\Phi(\mathbf{x}-\mathbf{y})=1$ for all $\mathbf{x}, \mathbf{y} \in T, \mathbf{x} \neq \mathbf{y}$, then $|T| \leq 2^{n}$, with equality iff $\left(\mathbb{R}^{n}, \Phi\right)$ is linearly isometric to $\ell_{\infty}^{n}$.

We will apply this theorem to the set $T=\left\{\sum_{i \in A} \mathbf{x}_{i} \mid A \subseteq\{1, \ldots, n\}\right\}$. Obviously $|T|=2^{n}$. We now show that

$$
\Phi\left(\sum_{i \in A} \mathbf{x}_{i}-\sum_{i \in B} \mathbf{x}_{i}\right)=1 \text { for all } A, B \subseteq\{1, \ldots, n\}, A \neq B,
$$

thus completing the proof. Firstly, we have

$$
\Phi\left(\sum_{i \in A} \mathbf{x}_{i}-\sum_{i \in B} \mathbf{x}_{i}\right)=\Phi\left(\sum_{i \in A \backslash B} \mathbf{x}_{i}+\sum_{i \in B \backslash A}-\mathbf{x}_{i}\right) \leq 1
$$

by $(A)$. Secondly, $A \backslash B \neq \emptyset$ or $B \backslash A \neq \emptyset$, since $A \neq B$. We assume without loss that $A \backslash B \neq \emptyset$ and choose $j \in A \backslash B$. Then

$$
\begin{aligned}
2=\Phi\left(2 \mathbf{x}_{j}\right) & \leq \Phi\left(\sum_{i \in A} \mathbf{x}_{i}-\sum_{i \in B} \mathbf{x}_{i}\right)+\Phi\left(\sum_{i \in B \cup\{j\}} \mathbf{x}_{i}-\sum_{i \in A \backslash\{j\}} \mathbf{x}_{i}\right) \\
& \leq \Phi\left(\sum_{i \in A} \mathbf{x}_{i}-\sum_{i \in B} \mathbf{x}_{i}\right)+1
\end{aligned}
$$

showing that (6) holds.

For strictly convex norms the bound in the above theorem should perhaps be $|S| \leq n+1$, but this seems to require a new idea.

\section{REFERENCES}

[BG] M. W. Bern and R. L. Graham, The shortest-network problem, Scientific American (January 1989), 66-71.

[BZ] Yu. D. Burago and V. A. Zalgaller, Geometric inequalities, Springer-Verlag, Berlin, Heidelberg, New York, 1988. MR 89b:52020

[C] J. A. Clarkson, Uniformly convex spaces, Trans. Amer. Math. Soc. 40 (1936), 396-414.

[CR] R. Courant and H. Robbins, What is Mathematics?, Oxford Univ. Press, Oxford, 1941. MR 3:144

[FLM] Z. Füredi, J. C. Lagarias and F. Morgan, Singularities of minimal surfaces and networks and related extremal problems in Minkowski space, Discrete and Computational Geometry (New Brunswick, NJ, 1989/1990), DIMACS Ser. Discrete Math. Theoret. Comput. Sci. 6, (J. E. Goodman, R. Pollack and W. Steiger, eds.), Amer. Math. Soc., Providence, RI, 1991, pp. 95-109. MR 93d:52009

[H] O. Hanner, On the uniform convexity of $L^{p}$ and $\ell^{p}$, Ark. Mat. 3 (1956), 239-244. MR 17:987 
[LM] G. Lawlor and F. Morgan, Paired calibrations applied to soap films, immiscible fluids, and surfaces and networks minimizing other norms, Pacific J. Math. 166 (1994), 55-82. MR 95i:58051

[M] F. Morgan, Minimal surfaces, crystals, networks, and undergraduate research, Math. Intelligencer 14 (1992), 37-44. MR 93h:53012

[Pe] C. M. Petty, Equilateral sets in Minkowski spaces, Proc. Amer. Math. Soc. 29 (1971), 369-374. MR 43:1051

[Pi] A. Pietsch, Operator Ideals, V. E. B. Deutscher Verlag Wiss., Berlin, 1978, and NorthHolland, Amsterdam, 1980. MR 81a:47002; MR 81j:47001

[vLW] J. H. van Lint and R. M. Wilson, A Course in Combinatorics, Cambridge Univ. Press, Cambridge, 1992. MR 94g:05003

Department of Mathematics and Applied Mathematics, University of Pretoria, PreTORIA 0002, SOUTH AFrica

E-mail address: konrad@friedrichs.up.ac.za 